\title{
Idiopathic chronic eosinophilic pneumonia and asthma: how do they influence each other?
}

\author{
E. Marchand*\#, B. Etienne-Mastroianni*, , P. Chanez*,+, D. Lauque*,§, P. Leclerc*,f, J.F. Cordier*, \\ and the Groupe d'Etudes et de Recherche sur les Maladies Orphelines Pulmonaires (GERM"O"P)
}

Idiopathic chronic eosinophilic pneumonia and asthma: how do they influence each other? E. Marchand, B. Etienne-Mastroianni, P. Chanez, D. Lauque, P. Leclerc, J.F. Cordier and the Groupe d'Etudes et de Recherche sur les Maladies Orphelines Pulmonaires (GERM"O"P). C) ERS Journals Ltd 2003.

ABSTRACT: Since idiopathic chronic eosinophilic pneumonia (ICEP) and asthma are frequently associated, their possible reciprocal influence on clinical presentation and evolution were investigated.

The clinical and follow-up features of 53 cases of ICEP, of which 41 (77\%) had asthma, were reviewed retrospectively. Asthma preceded the diagnosis of ICEP in 26 patients, was contemporaneous in eight patients, and developed $17 \pm 12$ months after ICEP in seven patients.

Presentation of ICEP was similar in asthmatics and nonasthmatics with the exception of a higher level of total immunoglobulin $\mathbf{E}$ in the former group. Patients with asthma at the time of diagnosis of ICEP were more likely to remain free of relapse of ICEP (56 versus 23\%) and had a lower number of relapses per year of follow-up (median 0 versus 0.24$)$. Moreover, they were treated more frequently with long-term inhaled corticosteroids (88 versus $31 \%$ ) at last follow-up. Asthma got worse after the diagnosis of ICEP and frequently required long-term oral corticosteroids.

To conclude, among patients with idiopathic chronic eosinophilic pneumonia, asthmatics have a lower frequency of relapse than nonasthmatics, possibly because of a higher use of inhaled corticosteroids. The occurrence of idiopathic chronic eosinophilic pneumonia in asthmatics is often associated with the development of severe asthma. Eur Respir J 2003; 22: 8-13.
*Centre d'Etudes et de Recherche sur les Maladies "Orphelines" Pulmonaires, Hôpital Cardiovasculaire et Pneumologique Louis Pradel, Université Claude Bernard, Hospices Civils de Lyon, "Service de Pneumologie, Hôpital Louis Pradel, Lyon, ${ }^{+}$Service de Pneumologie, Hôpital Arnaud de Villeneuve, Montpellier, ${ }^{\$}$ Service de Pneumologie, Centre Hospitalier Purpan, Toulouse and $f$ Service de Pneumologie, Centre Hospitalier, Sartrouville, France. ${ }^{\#}$ Service de Pneumologie, Cliniques Universitaires UCL de Mont-Godinne, Yvoir, Belgium.

Correspondence: J.F. Cordier, Hôpital Louis Pradel, BP Lyon-Montchat, 69394, Lyon cedex 03, France.

Fax: 33472357653

E-mail: germop@univ-lyon1.fr

Keywords: Asthma, follow-up studies, idiopathic chronic eosinophilic pneumonia, steroids

Received: September 162002

Accepted after revision: January 292003

This study was supported by grant HCLPHRC 93-97.005 from Ministère de 1'Emploi et de la Solidarité, Paris, France.
Idiopathic chronic eosinophilic pneumonia (ICEP) is a rare disorder of unknown cause characterised by subacute or chronic respiratory and general symptoms, alveolar and/or blood eosinophilia, and peripheral pulmonary infiltrates on chest imaging [1-3].

Airway eosinophilic infiltration is a hallmark of asthma [4]. Moreover, mild blood eosinophilia is sometimes present in asthmatic patients, and its level correlates with indices of clinical severity of the disease [5]. Interestingly, some but not all patients diagnosed with ICEP have a history of asthma $[2,3]$, whilst others may develop asthma after a diagnosis of ICEP has been made [3]. Response of ICEP to oral corticosteroid therapy is dramatic [1-3] but long-term oral corticosteroid therapy is necessary in up to one-half of the patients because of a high rate of relapses while decreasing or after stopping corticosteroid treatment [2,3]. The need for prolonged corticosteroid therapy not only stems from relapses of ICEP but also from the development of severe asthma [3].

The present study aimed to define better the reciprocal influence of asthma and ICEP, and particularly: 1) assess whether the presence of asthma at the time of diagnosis of ICEP influenced its presentation or was associated with a less favourable outcome in terms of relapses and the need for prolonged corticosteroid therapy; and 2) better determine the severity of asthma at the time of diagnosis of ICEP and its evolution thereafter.

\section{Methods}

\section{Recruitment of cases}

This study was undertaken by the Groupe d'Etudes et de Recherche sur les Maladies "Orphelines" Pulmonaires (GERM"O"P), a French collaborative group of pulmonary physicians dedicated to the study of rare (so-called "orphan") pulmonary diseases. Founded in 1993, the GERM"O"P includes over 200 physicians and 40 university hospitals mainly in France, but also in Belgium and Switzerland, with a coordination centre based in Lyon, France. Members keep in regular contact through newsletters and an annual meeting, and thus constitute a motivated and homogenous group. A registry of orphan pulmonary diseases has been implemented as a tool for clinical research. Starting from April 1995, participating physicians were requested to report all prevalent and incident cases of selected orphan pulmonary diseases to the registry, including ICEP. Reports were nominative for patients who 
gave written consent and anonymous in other cases. By June 2002, 1,435 cases of orphan pulmonary diseases had been reported to the registry.

\section{Data collection}

A detailed questionnaire was sent to all physicians having reported cases of ICEP to the registry. Questionnaires were completed by reviewing the medical records. Retrieved items included past medical history, medications, clinical and biological data, bronchoalveolar lavage (BAL), imaging (radiographs with or without high-resolution computed tomography), pulmonary function tests (PFTs), treatment and outcome. Special attention was paid to the number, timing and treatment of ICEP relapses. The diagnosis of asthma and its severity were assessed according to the Global Initiative for Asthma guidelines [6]. The timing of the diagnosis of asthma was also precisely reviewed. All previously published causes of eosinophilic pneumonias, such as drugs, allergic bronchopulmonary fungoses, parasitic and other infections were explicitly searched for throughout the questionnaire. A duration of symptoms of $>2$ weeks was required in order to distinguish ICEP from idiopathic acute eosinophilic pneumonia (IAEP) [7]. Signs of organ involvement outside the respiratory system were searched for in order to exclude patients with ChurgStrauss syndrome (CSS) or the idiopathic hypereosinophilic syndrome.

\section{Selection of cases}

All four of the following criteria were required for inclusion in the present study: 1) pulmonary infiltrates predominantly affecting the periphery of the lung on chest imaging; 2) blood $\left(\geqslant 1 \mathrm{~g} \cdot \mathrm{L}^{-1}\right)$ and/or alveolar ( $\geqslant 40 \%$ of BAL cell count) eosinophilia; 3 ) general and respiratory symptoms for $>2$ weeks; and 4) exclusion of known causes of eosinophilic pneumonia (especially drugs, parasitic infection, allergic bronchopulmonary funguses and CSS). Patients were excluded if they had signs of involvement of an extrarespiratory organ compatible with CSS and/or the idiopathic hypereosinophilic syndrome.

Relapses of ICEP were defined as the reappearance of characteristic new infiltrates on chest imaging, with compatible clinical features, and blood and/or BAL eosinophilia.

\section{Data analysis}

All questionnaires were carefully reviewed. The patients were divided into groups according to available data regarding the asthma status for analysis relevant in clinical practice. Accordingly, for analysis relevant to the diagnostic work-up (data at presentation), only patients known to have asthma before the diagnosis of ICEP were included in the group $(n=26)$ of "asthmatics prior to diagnosis of ICEP", and the other patients $(n=27)$ were included in the group of "nonasthmatics prior to diagnosis of ICEP". For analysis relevant to the long-term outcome, which is important to the clinician once the diagnosis of ICEP has been made, patients with a diagnosis of asthma made concurrently to that of ICEP were also included in a group called "asthmatics at the time of ICEP diagnosis". Moreover, only patients with a follow-up $>12$ months were included in the long-term analysis ("asthmatics at the time of diagnosis of ICEP", n=25; "nonasthmatics at the time of diagnosis of ICEP", $n=17$ ).

Doses of oral corticosteroids were expressed as prednisone equivalents. An obstructive pattern at PFTs was deemed present if the ratio forced expiratory volume in one second/ forced vital capacity was $<88 \%$ of predicted values and $<89 \%$ of predicted values in males and females, respectively. The predicted values for PFTs were those of QUANJER et al. [8]. Numerical data were expressed as mean \pm SD (as well as median in the absence of a Gaussian distribution), and compared by the two-way unpaired t-test or the Mann-Whitney test in the absence of a Gaussian distribution. Proportions were compared by the Chi-squared test or the Fisher's exact test, as required. The severity of asthma was studied by allocating a value of $0,1,2$ and 3 for remittent, mild-persistent, moderate-persistent and severe-persistent asthma, respectively. Comparison of asthma severity at the time of diagnosis of ICEP and at last follow-up was assessed using a Wilcoxon signed-rank test. For this particular analysis, only patients with a diagnosis of asthma prior to ICEP were included in the analysis. Indeed, it was judged that in a retrospective setting, the assessment of asthma severity was difficult without knowing the required chronic therapy. Statistical significance was defined as a p-value of $\leqslant 0.05$.

\section{Results}

A total of 70 questionnaires were sent to the centres having declared cases of ICEP on the GERM"O"P's registry. Of these, 55 filled-in questionnaires were received from 26 centres. Two questionnaires were rejected because of insufficient data to reliably exclude a known cause of eosinophilic pneumonia. Of the 53 selected questionnaires, 30 dealt with data from patients already included in a previous study on ICEP by the present group [3].

\section{Patients' characteristics at the time of diagnosis of idiopathic chronic eosinophilic pneumonia}

There were 34 females and 19 males (sex ratio: 1.8). The mean age was $43 \pm 17$ yrs. Only $13 \%$ of the patients were current smokers. Thirty-four $(64 \%)$ patients had a diagnosis of asthma by the time they were diagnosed as having ICEP, with the diagnosis of asthma and ICEP contemporaneous in eight of them. Seven patients $(13 \%)$ were diagnosed as having asthma after the diagnosis of ICEP (three at the time of an ICEP relapse), $17 \pm 10$ months after the diagnosis of ICEP. Only $12(23 \%)$ patients never had asthma.

The mean time interval between the onset of symptoms and the diagnosis of ICEP was $31 \pm 69$ weeks (median 8 weeks). Blood eosinophilia at diagnosis of ICEP was $4.61 \pm 5.11 \mathrm{~g} \cdot \mathrm{L}^{-1}$ (median $2.99 \mathrm{~g} \cdot \mathrm{L}^{-1}$ ), which represented $30.8 \pm 15.3 \%$ of the total leukocyte count. Increased levels of total immunoglobulin (Ig)E $\left(>120\right.$ International Units (IU) $\left.\mathrm{mL}^{-1}\right)$ were found in $67 \%$ of the patients. The total IgE level was $499 \pm 620 \mathrm{IU} \cdot \mathrm{mL}^{-1}$ (median $\left.208 \mathrm{IU} \cdot \mathrm{mL}^{-1}\right)$. The cellular differential count in the BAL $(\mathrm{n}=28)$ revealed $54 \pm 25 \%$ eosinophils (macrophages $35 \pm 20 \%$, lymphocytes $6 \pm 5 \%$, neutrophils $5 \pm 6 \%$ ). An obstructive pattern was found in $43 \%$ of the cases on PFTs at diagnosis of ICEP.

When comparing asthmatics and nonasthmatics prior to diagnosis of ICEP, there were no significant differences between the groups for the above-mentioned characteristics, with the exception of a higher level of blood total IgE (median 425 versus $128 \mathrm{IU} \cdot \mathrm{mL}^{-1}, \mathrm{p}=0.03$, Mann-Whitney test; table 1) in asthmatics prior to diagnosis of ICEP. Moreover, there was a tendency for a lower level of BAL eosinophilia in asthmatics prior to diagnosis of ICEP, with the same tendency observed when comparing patients with or without inhaled corticosteroids at the time of diagnosis of ICEP $(p=0.08)$. 
Table 1. - Patients' characteristics at the time of diagnosis of idiopathic chronic eosinophilic pneumonia (ICEP)

\begin{tabular}{|c|c|c|c|c|}
\hline & All patients & Asthmatics & Nonasthmatics & p-value \\
\hline Age yrs & $43 \pm 17$ & $44 \pm 18$ & $41 \pm 17$ & 0.61 \\
\hline $\mathrm{M} / \mathrm{F}$ & $19 / 34$ & $12 / 14$ & $7 / 20$ & 0.21 \\
\hline Current smokers \% & 13 & 5 & 20 & 0.19 \\
\hline Delay of diagnosis ${ }^{\#}$ weeks & $31 \pm 69$ & $43 \pm 85(8)$ & $19 \pm 46(7)$ & 0.33 \\
\hline Blood eosinophilia $\mathrm{g} \cdot \mathrm{L}^{-1}$ & $4.6 \pm 5.1$ & $4.3 \pm 5.3(2.8)$ & $4.8 \pm 5(3.1)$ & 0.46 \\
\hline Blood eosinophilia $\%$ leukocytes & $31 \pm 15$ & $29 \pm 16(25)$ & $32 \pm 15(30)$ & 0.37 \\
\hline Total IgE IU $\cdot \mathrm{mL}^{-1}$ & $499 \pm 620(208)$ & $693 \pm 750(425)$ & $304 \pm 386(128)$ & 0.03 \\
\hline ESR $\mathrm{mm} \cdot \mathrm{h}^{-1}$ & $65 \pm 33$ & $61 \pm 31$ & $68 \pm 35$ & 0.48 \\
\hline BAL eosinophilia $\%$ tcc & $54 \pm 25$ & $45 \pm 26$ & $61 \pm 22$ & 0.08 \\
\hline With bilateral infiltrates on radiographs $\%$ & 83 & 71 & 92 & 0.11 \\
\hline FEV1 $\%$ pred & $71 \pm 23$ & $69 \pm 22$ & $73 \pm 25$ & 0.6 \\
\hline With obstructive PFT \% & 43 & 54 & 29 & 0.15 \\
\hline
\end{tabular}

Data are presented as mean \pm SD (median) unless otherwise stated. Asthmatics: patients with a diagnosis of asthma prior to ICEP; Nonasthmatics: patients without a diagnosis of asthma prior to ICEP; M: male; F: female; Ig: immunoglobulin; IU: International Units; ESR: erythrocyte sedimentation rate; BAL: bronchoalveolar lavage; tcc: total cell count; FEV1: forced expiratory volume in one second; \% pred: per cent predicted; PFT: pulmonary function test. ${ }^{\#}$ : time between onset of symptoms and diagnosis of ICEP; ${ }^{\uparrow}$ : BAL data were available in 28 patients (15 without and 13 with asthma before ICEP, respectively).

\section{Characteristics of asthma at the time of diagnosis of idiopathic chronic eosinophilic pneumonia}

Of the 26 patients $(50 \%)$ with a diagnosis of asthma prior to that of ICEP, the time interval between the diagnosis of the two diseases was $74 \pm 96$ months (median 42 months). Twentythree, 14 and eight patients were asthmatics for $>1,>3$ and $>5$ yrs, respectively. The severity of asthma was classified as mild persistent, moderate persistent and severe persistent in nine, 12 and four patients, respectively. One patient with a prior diagnosis of asthma was considered to have remittent disease at the diagnosis of ICEP. Of the asthmatic patients, $60 \%$ had already needed oral corticosteroid therapy because of an acute exacerbation of asthma.

\section{Long-term outcome}

The analysis of the long-term outcome was restricted to the 42 patients followed for $>12$ months after the diagnosis of ICEP (mean follow-up $87 \pm 62$ months, median 63 months). Among these patients, a diagnosis of asthma was made before, at the same time as or after the diagnosis of ICEP in 20 , eight and four patients, respectively. Ten patients followed for $>12$ months never developed asthma.

Relapses of idiopathic chronic eosinophilic pneumonia. Relapses occurred in 24 patients $(57 \%)$ with a mean of $2.3 \pm 1.4$ relapses (median 2 relapses) during the follow-up or $0.44 \pm 0.57$ relapses $\cdot y r$ of follow-up ${ }^{-1}$ (YFU; median 0.32 relapses $\cdot \mathrm{YFU}^{-1}$ ).

Asthmatics at the time of diagnosis of ICEP had fewer relapses during the follow-up compared with nonasthmatics at the time of diagnosis of ICEP. This was true whether expressed in total number of relapses $(0.9 \pm 1.2$ (median 0 ) versus $1.9 \pm 1.7$ (median 1.0) relapses, $\mathrm{p}<0.05$, Mann-Whitney test) or in number of relapses. YFU ${ }^{-1}(0.14 \pm 0.2$ (median 0$)$ versus $0.42 \pm 0.7$ (median 0.24 ) relapses $\cdot \mathrm{YFU}^{-1}, \mathrm{p}<0.05$, MannWhitney test; fig. 1a). More asthmatics than nonasthmatics at the time of diagnosis of ICEP never experienced any relapse during the follow-up (56 versus $23 \%, \mathrm{p}<0.05$, Fisher's exact test), the length of follow-up being similar in the two groups ( $88 \pm 66$ versus $84 \pm 60$ months, $\mathrm{p}=0.97$, Mann-Whitney test).

At the last follow-up visit, $65 \%$ of the patients were on long-term inhaled corticosteroids and $56 \%$ were still on oral corticosteroid therapy. The reason for long-term inhaled therapy was asthma, except in one patient for whom it was initiated for preventing relapses of ICEP. More patients with than without a diagnosis of asthma at the time of diagnosis of ICEP were on long-term inhaled corticosteroids (88 versus $31 \%, \mathrm{p}<0.001$, Fisher's exact test). Patients on long-term inhaled corticosteroids also had fewer relapses $(0.14 \pm 0.16$ (median 0.04 ) versus $0.51 \pm 0.76$ (median 0.33 ) relapses $\cdot \mathrm{YFU}^{-1}$, $\mathrm{p}<0.05$, Mann-Whitney test; fig. 1b). The inhaled corticosteroids used by the patients in the present study were distributed among beclomethasone $(47 \%)$, budesonide (21\%) and fluticasone $(32 \%)$. Doses varied from $500 \mu \mathrm{g} \cdot \mathrm{day}^{-1}$ of beclomethasone to a maximum of $3 \mathrm{mg} \cdot \mathrm{day}^{-1}$ of beclomethasone or $2 \mathrm{mg} \cdot \mathrm{day}^{-1}$ of fluticasone.

The reason for pursuing oral corticosteroids was asthma in $61 \%$ and multiple ICEP relapses in $39 \%$ of the cases. There were no significant differences in the number of relapses in patients with or without oral corticosteroid therapy at the last follow-up $(0.27 \pm 0.61$ (median 0.07 ) versus $0.25 \pm 0.29$ (median $0.17)$ relapses. $\left.\mathrm{YFU}^{-1}\right)$.

Among the $43 \%$ of patients without ICEP relapse, $81 \%$ were on long-term inhaled corticosteroids and $53 \%$ were on oral corticosteroids because of asthma at last follow-up visit.

Outcome of asthma. In the 20 patients with a diagnosis of asthma prior to that of ICEP, all those initially treated with inhaled corticosteroids were still on this type of therapy at the last follow-up visit. Six of the eight asthmatics, not initially treated with inhaled corticosteroids, were receiving this type of medication at the last visit. The same pattern of evolution was observed for oral corticosteroids with the two patients initially on treatment (dose 20 and $5 \mathrm{mg} \cdot$ day $^{-1}$, respectively) still taking oral corticosteroids at the last control (dose 40 and $12.5 \mathrm{mg}$. day $^{-1}$, respectively) and six $(33 \%)$ of the patients not initially treated with oral corticosteroids receiving $17 \pm 19 \mathrm{mg} \cdot$ day $^{-1}$ prednisone (median 14) because of asthma at the last visit. The severity of asthma significantly increased after ICEP, as shown in figure 2 .

Of the 12 patients diagnosed with asthma at the time of or after the diagnosis of ICEP, eight $(67 \%)$ and five $(42 \%)$ were treated with inhaled and oral corticosteroids $\left(5 \pm 3 \mathrm{mg} \cdot \mathrm{day}^{-1}\right.$ prednisone (median 4.5)) at the last follow-up visit, respectively.

\section{Discussion}

The main results of the present study are three-fold. First, the study highlighted the fact that ICEP may occur in some patients as a rare complication of asthma, although it is seldom 

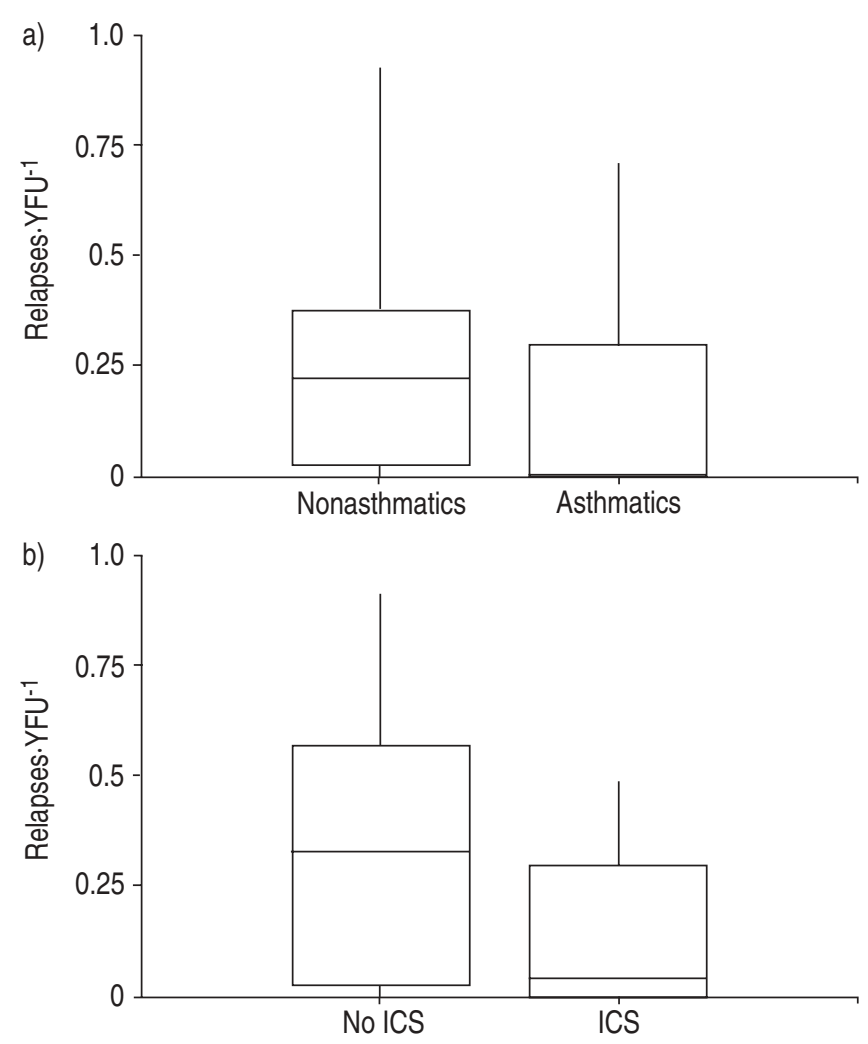

Fig. 1.-Percentile plots of the number of relapses per year of follow-up (YFU) showing a) a lower frequency of relapses in asthmatics versus nonasthmatics at the time of idiopathic chronic eosinophilic pneumonia diagnosis $(\mathrm{p}=0.04)$ and $\mathrm{b})$ in patients with versus without long-term inhaled corticosteroids (ICS) therapy $(\mathrm{p}=0.02)$. The lower and the upper limits of the boxes represent the 25th and the 75th percentiles, respectively. The horizontal lines represent the medians and the upper limits of the vertical lines represent the maximum values.

mentioned in reviews and textbooks on asthma. Secondly, when present in patients with ICEP, asthma was relatively severe and got worse after the diagnosis of ICEP. Thirdly, the presence of asthma at the time of diagnosis of ICEP was associated with less relapses of ICEP, possibly because of a higher frequency of long-term inhaled corticosteroids use in asthmatics.

The prevalence of asthma in patients diagnosed with ICEP in this study $(64 \%)$ is higher than reported in the literature previously [2, 3, 9-12], from 28-53\% [1]. The first studies of ICEP suggested that when present at the time of diagnosis of ICEP, asthma was of recent onset $(<1 \mathrm{yr})[1,9,10]$. However, in line with other series $[2,12]$, it was found that the time interval between the diagnosis of asthma and ICEP can be much longer, with a median of $>3$ yrs. Interestingly, as reported previously by DURIEU et al. [13], as well as the present group [3], the current study confirms that asthma may develop concurrently to or after the diagnosis of ICEP.

The association of ICEP and asthma may be regarded as rather logical and even expected, since both conditions are associated with eosinophilic infiltration of the respiratory tract. However, $23 \%$ of the patients in the present study never developed asthma in the follow-up. Furthermore, the authors reported the presence of eosinophilic airway infiltration demonstrated by bronchial biopsies in three patients with ICEP without a previous history of asthma [3]. This parallels other conditions associated with eosinophilic inflammation involving the respiratory tract that are not associated with asthma. For example, neither IAEP [7, 14] nor eosinophilic

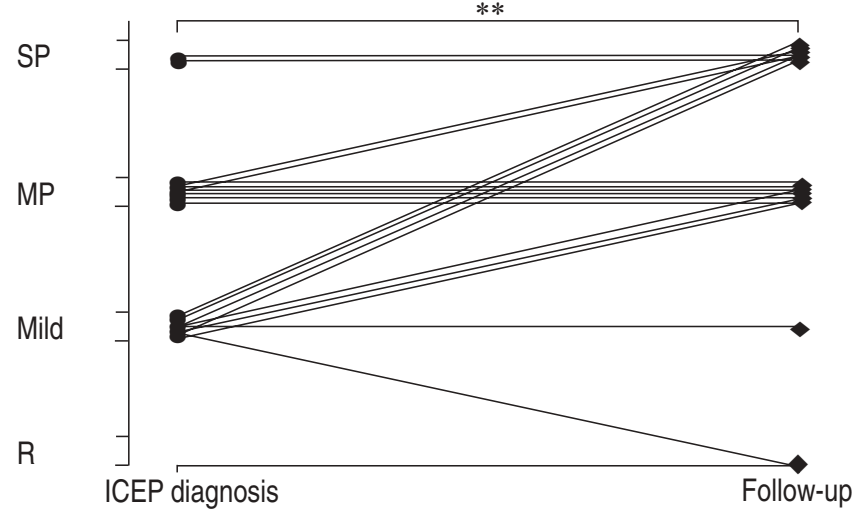

Fig. 2.-Asthma severity at the time of diagnosis of idiopathic chronic eosinophilic pneumonia (ICEP) and at the time of last follow-up visit in the 20 patients with a diagnosis of asthma preceding ICEP followed for $>1 \mathrm{yr}$. The severity of asthma was assessed according to the Global Initiative for Asthma guidelines [6] with a value of $0,1,2$ and 3 assigned to remittent (R), mild persistent (Mild), moderate persistent (MP) and severe persistent (SP) asthma, respectively. Asthma severity at last follow-up was increased compared with asthma severity at the time of diagnosis of ICEP diagnosis. $* *$ : $<<0.01$, Wilcoxon signed-rank test.

bronchitis $[15,16]$ have been reported to be associated with asthma. By separating patients according to the presence or not of asthma at the time of diagnosis, one of the aims was to define two different subsets of patients with ICEP. It turned out that there were indeed differences between these two groups. However, as discussed below, the main discrepancies dealt with the long-term outcome of the disease and can potentially be explained by differences in the treatment strategies.

The present study also demonstrates that asthma gets worse after the diagnosis of ICEP. Mild blood eosinophilia is present in some asthmatic patients and it is known that its level correlates with indices of clinical severity of the disease [5]. Accordingly, the development of ICEP in asthmatics may be understood as a step towards more severe disease, including a worsening of the airway eosinophilic disease and the development of parenchymal eosinophilic infiltration.

The severity of asthma associated with ICEP is also underlined by the fact that more than one-half of the asthmatic patients required long-term oral corticosteroids at the last follow-up visit. This compares to a $5 \%$ proportion of patients requiring oral corticosteroids in a large French crosssectional study of asthmatics attending private-practice chest specialists [17]. Accordingly, asthma in patients with ICEP must be considered to be much more severe than in the general population.

An aim of the present study was to examine whether the presence of asthma at diagnosis of ICEP had an influence on the long-term outcome of the latter. The authors' initial hypothesis was that asthma could be associated with a worse prognosis of ICEP. Indeed, CSS, another eosinophilic disease with systemic involvement associated with asthma that may have close links with ICEP [3], is associated with relapses [18]. Conversely, IAEP is not associated with asthma and does not relapse [7]. Contrary to the initial hypothesis, the authors observed a lower number of relapses of ICEP among asthmatics at the time of diagnosis of ICEP. Moreover, more patients initially presenting with asthma never experienced any relapse. As expected, more patients with initial asthma were on long-term inhaled corticosteroids at the last followup visit. Together with the presence of asthma at the time of diagnosis of ICEP, long-term use of inhaled corticosteroids was the only parameter associated with fewer ICEP relapses. In addition, asthmatics at the time of diagnosis of ICEP also 
tended to have a lower BAL eosinophilia and a lower frequency of bilateral involvement on chest imaging. The same tendency was observed for patients on inhaled corticosteroids at the time of diagnosis of ICEP. This observation suggests that inhaled corticosteroids may decrease or control manifestations of ICEP.

These findings can be put in parallel with the development of CSS in association with specific asthma therapies. It appears that in the vast majority, CSS developed during decreasing corticosteroid usage [19], suggesting that corticosteroids could have controlled CSS to some extent. Since corticosteroids represented the main treatment of the asthmatic patients included in the present study, the data suggest that the same may be true for ICEP.

A role for inhaled corticosteroid treatment in ICEP has already been suggested by NAUGHTON et al. [12] and LAVANDIER and CARRE [20], who proposed that these would allow a decrease in doses of systemic corticosteroids in patients requiring prolonged therapy. The present study provides further evidence for a possible role of inhaled corticosteroids in preventing relapses of ICEP. The inhaled corticosteroids used by the patients in the present study were distributed among beclomethasone, budesonide and fluticasone. The small number of patients and the various doses used prevented conclusions from being drawn regarding the putative superiority of a particular type or dose of inhaled corticosteroid.

Of the patients in the present study, $43 \%$ did not present any ICEP relapse during the follow-up, and thus it could be argued that ICEP has a good prognosis in a large proportion of cases. However, less than one-quarter of the patients diagnosed with ICEP have a simple outcome, i.e. without ICEP relapse and/or the need for prolonged oral corticosteroid therapy for asthma.

Two findings related to putative differences between asthmatics and nonasthmatics deserve further discussion. First, though not significantly different from the nonasthmatics, the sex ratio of asthmatics prior to diagnosis of ICEP was near to one. This is unusual in ICEP, which is more frequent in females [1-3]. The authors have no explanation for this finding. Secondly, the prevalence of smoking tended to be higher in nonasthmatics prior to diagnosis of ICEP. This may be due to the fact that asthmatics are more likely to quit smoking than nonasthmatics. However, the fact that smoking may promote the development of asthma in patients who will develop ICEP cannot be excluded. Moreover, this observation should not be overemphasised, since the number of smokers was very small, which is usual in ICEP [2, 3].

The present study has some limitations mainly due to its retrospective character. Although all episodes of ICEP were treated with oral corticosteroids in all patients included in the present study, the doses and duration were not uniform and could thus influence the risk of relapse occurrence. However, there are no data in the literature to show that either the dosage or the duration of oral corticosteroids have any influence on the risk of ICEP relapse. Prospective studies addressing this issue are difficult to perform in ICEP because of its rarity, even by collaborative groups such as the GERM"O"P.

To conclude, the present study confirms the strong association between idiopathic chronic eosinophilic pneumonia and asthma, and the influence of both diseases on each other. Patients that had asthma at the time of diagnosis of idiopathic chronic eosinophilic pneumonia had less frequent idiopathic chronic eosinophilic pneumonia relapses, which may suggest that inhaled corticosteroids have a favourable influence on the course of idiopathic chronic eosinophilic pneumonia. However, idiopathic chronic eosinophilic pneumonia is often associated with severe and worsening asthma, with more than one-third of the patients eventually requiring

long-term oral corticosteroid therapy because of severe asthma.

\begin{abstract}
Members of the GERM"O"P participating in the study: France: E. Bidat (Paris), J.M. Bréchot (Paris), P.M. Broussier (Bois-Guillaume), J. Charbonneau (Montbéliard), C. Claude (Trégastel), D. Coëtmeur (Saint Brieuc), J.F. Cordier (Lyon), B. Crestani (Paris), P. Delaval (Rennes), A. Dietemann (Strasbourg), P. Dore (La Rochelle), C. Eveilleau (Brest), P. Girard (Tassin), J.Y. Jasnot (Nantes), D. Lauque (Toulouse), P. Leclerc (Sartrouville), C. Leroyer (Brest), P. Ode (Saint Etienne), F. Philip-Joet (Marseille), C. Pison (Grenoble), L. Sohier (Lorient), E. Tuchais (Angers), A. Vandevenne (Strasbourg), G. Vernet (Le Puy en Velay), P. Chanez (Montpellier). Belgium: L. Delaunois (Yvoir)
\end{abstract}

\section{References}

1. Carrington $\mathrm{CB}$, Addington WW, Goff AM, et al. Chronic eosinophilic pneumonia. $N$ Engl J Med 1969; 280: 787-798.

2. Jederlinic PJ, Sicilian L, Gaensler EA. Chronic eosinophilic pneumonia. A report of 19 cases and a review of the literature. Medicine (Baltimore) 1988; 67: 154-162.

3. Marchand E, Reynaud-Gaubert M, Lauque D, Durieu J, Tonnel AB, Cordier JF. Idiopathic chronic eosinophilic pneumonia. A clinical and follow-up study of 62 cases. The Groupe d'Etudes et de Recherche sur les Maladies "Orphelines" Pulmonaires (GERM"O"P). Medicine (Baltimore) 1998; 77: 299-312.

4. Lacoste JY, Bousquet J, Chanez P, et al. Eosinophilic and neutrophilic inflammation in asthma, chronic bronchitis, and chronic obstructive pulmonary disease. J Allergy Clin Immunol 1993; 92: 537-548.

5. Bousquet $\mathrm{J}$, Chanez $\mathrm{P}$, Lacoste JY, et al. Eosinophilic inflammation in asthma. N Engl J Med 1990; 323: 10331039.

6. National Heart, Lung and Blood Institute. Global strategy for asthma management and prevention. Updated from NIH publication no. 02-3659. Bethesda, National Institutes of Health, 2002; pp. 1-176.

7. Pope-Harman AL, Davis WB, Allen ED, Christoforidis AJ, Allen JN. Acute eosinophilic pneumonia. A summary of 15 cases and review of the literature. Medicine (Baltimore) 1996; 75: 334-342.

8. Quanjer PH, Tammeling GJ, Cotes JE, Pedersen OF, Peslin R, Yernault JC. Lung volumes and forced ventilatory flows. Report Working Party Standardization of Lung Function Tests, European Community for Steel and Coal. Official Statement of the European Respiratory Society. Eur Respir J 1993; 6: Suppl. 16, 5s-40s.

9. Gaensler EA, Carrington CB. Peripheral opacities in chronic eosinophilic pneumonia: the photographic negative of pulmonary edema. AJR Am J Roentgenol 1977; 128: 1-13.

10. Pearson DL, Rosenow EC 3rd. Chronic eosinophilic pneumonia (Carrington's): a follow-up study. Mayo Clin Proc 1978; 53: 73-78.

11. Bancal C, Sadoun D, Valeyre D, et al. Pneumopathie chronique idiopathique à éosinophiles. Presse Med 1989; 18: $1695-1698$.

12. Naughton M, Fahy J, Fitzgerald MX. Chronic eosinophilic pneumonia. A long-term follow-up of 12 patients. Chest 1993; 103: 162-165.

13. Durieu J, Wallaert B, Tonnel AB. Long-term follow-up of pulmonary function in chronic eosinophilic pneumonia. Groupe d'Etude en Pathologie Interstitielle de la Societe de Pathologie Thoracique du Nord. Eur Respir J 1997; 10: 286-291.

14. Allen JN, Pacht ER, Gadek JE, Davis WB. Acute 
eosinophilic pneumonia as a reversible cause of noninfectious respiratory failure. $N$ Engl J Med 1989; 321: 569-574.

15. Gibson PG, Dolovich J, Denburg J, Ramsdale EH, Hargreave FE. Chronic cough: eosinophilic bronchitis without asthma. Lancet 1989; 1: 1346-1348.

16. Brightling CE, Ward R, Goh KL, Wardlaw AJ, Pavord ID. Eosinophilic bronchitis is an important cause of chronic cough. Am J Respir Crit Care Med 1999; 160: 406-410.

17. Liard R, Leynaert B, Zureik M, Beguin FX, Neukirch F. Using Global Initiative for Asthma guidelines to assess asthma severity in populations. Eur Respir J 2000; 16: 615-620.
18. Guillevin L, Cohen P, Gayraud M, Lhote F, Jarrousse B Casassus P. Churg-Strauss syndrome. Clinical study and long-term follow-up of 96 patients. Medicine (Baltimore) 1999; 78: 26-37.

19. Weller PF, Plaut M, Taggart V, Trontell A. The relationship of asthma therapy and Churg-Strauss syndrome: NIH workshop summary report. J Allergy Clin Immunol 2001; 108: 175-183.

20. Lavandier M, Carre P. Effectiveness of inhaled high-dose corticosteroid therapy in chronic eosinophilic pneumonia. Chest 1994; 105: 1913-1914. 